\title{
Elaboración del Informe Médico Legal de Lesiones Odontológico: Recomendaciones para el Odontólogo
}

\author{
Elaboration of the Dental Legal Report of Dental Injuries: \\ Recommendations for the Dentist
}

Denisse Lagos Tissie ${ }^{1,2,3}$ \& Lidia Bravo R.,

LAGOS, T. D. \& BRAVO, R. L. Elaboración del informe médicolegal de lesiones odontológico: recomendaciones para el odontólogo. Int. J. Odontostomat., 13(3):350-356, 2019.

RESUMEN: El odontólogo juega un papel relevante en la acreditación o constatación de lesiones, especialmente si cumple labores en servicios de urgencia. Esta primera evaluación es fundamental para que el perito en lesionología forense del Servicio Médico Legal (SML) se pronuncie posteriormente respecto del pronóstico médico legal de las lesiones, señalando el tiempo que demorarán en sanar o la incapacidad laboral que generan, además de vincularlas causalmente con los hechos delictivos investigados. Se presenta información actualizada sobre el proceso de constatación de lesiones, el informe de lesiones en la legislación chilena y la participación que le cabe al cirujano dentista. La preparación de un correcto informe médicolegal de lesiones es un acto médico que no se puede delegar a otro miembro del personal de salud que no sea el profesional médico tratante. Todo cirujano dentista debe tener la capacidad de generar un primer informe de lesiones apropiado a las exigencias legales y éticas que la sociedad exige. La ley expresa la posibilidad de citar a un juicio oral, en calidad de testigo, al profesional que haya confeccionado un informe médicolegal de lesiones (constatación de lesiones), con el propósito de dar testimonio de manera directa, explicando desde un punto de vista profesional lo registrado.

PALABRAS CLAVE: odontología forense; legislación; lesionología maxilofacial.

\section{INTRODUCCIÓN}

El odontólogo juega un papel relevante en la acreditación o constatación de lesiones, especialmente si cumple labores en servicios de urgencia, tanto públicos como privados. En ellos, frecuentemente se verá enfrentado a personas que presenten lesiones físicas en el territorio maxilofacial, ya sea de carácter accidental (caídas, hechos de tránsito) como intencional (riñas, violencia intrafamiliar), y requerirá de sus conocimientos expertos para constatarlas como indica la ley.

Esta primera evaluación es fundamental para que el perito en lesionología forense del Servicio Médico Legal (SML) se pronuncie posteriormente respecto del pronóstico médico legal de las lesiones, señalando el tiempo que demorarán en sanar o la incapacidad laboral que generan, además de vincularlas causalmente con los hechos delictivos investigados.
El objetivo de este trabajo es presentar información actualizada sobre el proceso de constatación de lesiones, el informe de lesiones en la legislación chilena y la participación que le cabe al cirujano dentista.

Marco Legal. Si bien muchas lesiones responden a accidentes de la vida cotidiana, otras tienen su origen en situaciones que merecen una investigación judicial: lesiones causadas por terceros (riñas, asaltos, violencia intrafamiliar, maltrato infantil, etc.), lesiones autoinferidas (simulaciones, suicidios), lesiones accidentales (accidentes de tránsito, en el lugar de trabajo o en el domicilio). Las lesiones deben despertarnos sospecha especialmente cuando su manifestación clínica no coincide con el relato del paciente o su acompañante.

\footnotetext{
${ }^{1}$ Especialista en Odontología Legal y Forense.

${ }^{2}$ Medicina Legal Odontológica, Facultad de Odontología, Universidad de Chile, Santiago, Chile.

${ }^{3}$ Odontología Legal, Carrera de Odontología, Escuela de Ciencias de la Salud, Universidad Viña del Mar, Viña del Mar, Chile.
} 
En general, el ordenamiento jurídico chileno obliga a los jefes de establecimientos hospitalarios o de clínicas particulares, a los profesionales en medicina, odontología, química y farmacia, y de otras ramas relacionadas con la conservación o el restablecimiento de la salud, a denunciar a las autoridades cuando se enfrenten a cualquier individuo que tuviere lesiones corporales de significación. En la denuncia debe constar el estado del paciente, la descripción de los signos externos de las lesiones e incluir las explicaciones que dieran el afectado o las personas que lo hubieren conducido (art. 175 y 200 del CPP) (Fiscalía, Ministerio Público de Chile, 2000). El incumplimiento del deber de denuncia por parte del profesional lo hace incurrir en una falta, cuya pena corresponde a una multa de 1 a 4 Unidades Tributarias Mensuales, según dispone el artículo 494 del Código Penal (Fiscalía, Ministerio Público de Chile, 2018).

Sumado a lo anterior, cabe añadir que algunas lesiones son sancionadas de acuerdo a figuras delictivas que la jurisprudencia considera más graves, $y$, por tanto, su falta de registro y/o denuncia por parte del profesional médico implican una sanción para él, pues deja en desprotección a la víctima. Estas figuras son:

- Violencia intrafamiliar: La Ley 20.066, en su art. 21 letra d, indica que "en ningún caso el tribunal podrá calificar como leves las lesiones cometidas en contra de las personas mencionadas en el artículo $5^{\circ}$ de la Ley sobre Violencia Intrafamiliar" (Ministerio de Justicia, 2005).

- Accidentes de tránsito: La Ley 18.290 en su art. 196e, indica que "se reputarán leves, para estos efectos, todas las lesiones que produzcan al ofendido enfermedad o incapacidad por un tiempo no mayor de siete días" (Ministerio de Justicia, 1984).

- Abuso sexual. El Código Procesal Penal, en su art. 198, indica que "los hospitales, clínicas y establecimientos de salud semejantes, sean públicos o privados, deberán practicar los reconocimientos, exámenes médicos y pruebas biológicas conducentes a acreditar el hecho punible y a identificar a los partícipes en su comisión, debiendo conservar los antecedentes y muestras correspondientes" (Código Procesal Penal).

- Accidentes del Trabajo y Enfermedades Profesionales: En su art.76 señala que "El accidentado o enfermo, o sus derecho-habientes, o el médico que trató o diagnosticó la lesión o enfermedad, como igualmente el Comité Paritario de Seguridad, tendrán, también, la obligación de denunciar el hecho en dicho organismo administrador, en el caso de que la entidad empleadora no hubiere realizado la denuncia" (Ministerio del Trabajo y Previsión Social, 1984).

Informe médicolegal de lesiones. La preparación de una correcta constatación de lesiones, o informe médicolegal de lesiones, es un acto médico que no se puede delegar a otro miembro del personal de salud que no sea el profesional médico tratante (médico cirujano o cirujano dentista, general o especialista). En los servicios de atención de urgencia, la constatación de lesiones se realiza en el Dato de Atención de Urgencia (DAU). El Decreto Exento 467/ 13 del Ministerio de Salud, que aprueba la Norma Técnica 149, establece las variables que constituyen el conjunto mínimo básico de datos que éste debe contener. Algunas de estas variables son datos estadísticos, como lo relativo a la identificación del paciente (nombre, edad, RUN, fecha de nacimiento, sexo, previsión, teléfono, dirección, fecha y hora de ingreso), el tipo de accidente que motiva la consulta (de trabajo, escolar, de trayecto, agresión, tránsito, violencia de género, etc), su lugar de ocurrencia y el medio de transporte en que fue trasladado hasta el Servicio de Urgencia. Al ser ingresado al servicio, se le realizará una categorización, que corresponde a un ordenamiento de la atención de los consultantes en un servicio de urgencia, el cual es realizado exclusivamente por enfermería (emergencia vital, emergencia evidente, urgencia médica relativa, urgencia potencial, atención médica general) (Ministerio de Salud, 2013) (Fig. 1).

La Norma exige que en el DAU conste, como mínimo, el motivo de consulta del paciente, la individualización de todo el personal médico y no médico que realice evaluación del paciente; la hipótesis diagnóstica inicial y a la final del tratamiento, el pronóstico médico legal de las lesiones y todas las indicaciones dadas por los profesionales tanto en la estadía en el Servicio como las indicaciones al alta.

Si bien la Norma Técnica 149 operacionaliza el Decreto 467/13 y establece un estándar de confección y llenado del DAU a seguir por los servicios públicos y privados del país, habitualmente encontramos que en algunos servicios se incluyen otras secciones en este documento, como son "origen de las lesiones según relato" y "origen de las lesiones según la apreciación clínica", además de disponer de un espacio 


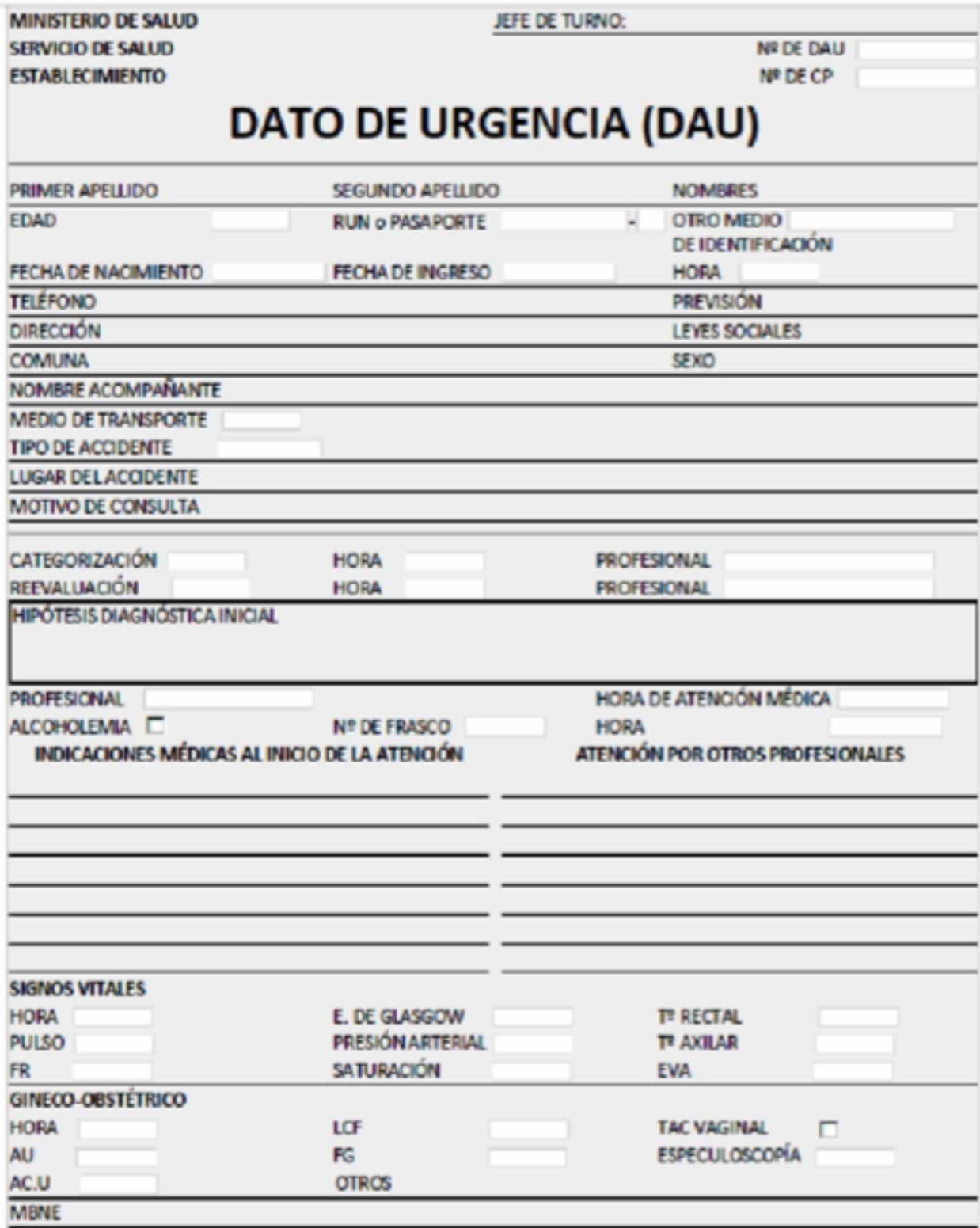

EOOGRAFA

\begin{tabular}{|c|c|}
\hline DESTINO & PRONUĆS nCO MÉD. IFCAL PROMISORO. \\
\hline
\end{tabular}

\begin{tabular}{|c|c|c|}
\hline \multicolumn{3}{|c|}{ HIDOTESIS DIAGNOSTICAAL. TERRMINO DE LA ATENCIÓN DE URGENCIA } \\
\hline \multicolumn{3}{|c|}{ INDICAOONES AL TÉRMINO DE LA ATENCOON } \\
\hline MEOICO QUE AUTORZA EL ALTA & RUN & 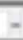 \\
\hline
\end{tabular}

Lo hipótesis dogonóstico (o diognóstike) es provisoria. pues puede sufrir alterociones debidas a lo evolución de ie

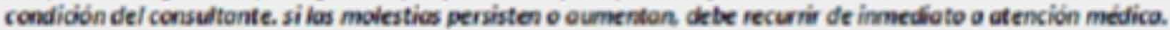

Fig. 1. Documento propuesta base DAU, Decreto Exento 467/13 del Ministerio de Salud. mayor para la descripción y diagnóstico de la lesión que justifique la hipótesis diagnóstica.

Origen de la lesión según relato. En la anamnesis, se debe orientar al paciente a que relate las circunstancias en que ocurrieron los hechos, el agente causal de las lesiones y su mecanismo de acción. De ser necesario, esta información se puede citar entre comillas. El profesional debe ser capaz de detectar contradicciones o confusiones en el relato que aumenten o minimicen la gravedad de los hechos, o intenten crear situaciones falsas, de modo de correlacionarlo con lo que observa clínicamente.

Origen de la lesión según la apreciación clínica. La explicación profesional sobre cómo se produjo la lesión es el resultado de la ponderación de la anamnesis, del examen físico extra e intraoral y del conocimiento del profesional sobre patrones lesionales, considerando los hallazgos clínicos y también la ausencia de hallazgos (información negativa) (Bórquez, 2012). Estos patrones lesionales hacen referencia a la ubicación anatómica de las lesiones en distintos contextos o situaciones:

- Agresión: en estos casos, se aprecian lesiones en nudillos de manos y en la cara anterior de piernas y pies. 
- Defensa: lesiones en las manos (especialmente en los pliegues en un intento de agarrar el objeto que ocupa el agresor) y en los antebrazos (especialmente en las caras laterales y su cara posterior, en un intento reflejo de proteger la cara del agresor).

- Inmovilización: lesiones en muñecas, tobillos, piel perioral, cara interna de mejillas y mucosa intraoral.

- Lucha: partes salientes del rostro, cuero cabelludo, nudillos y extremidades en general.

Como se puede apreciar, estos patrones de distribución consideran áreas anatómicas que no son del territorio maxilofacial. Si bien el cirujano dentista debe dar su opinión experta en el área que es de su competencia, no puede dejar de lado la observación del resto del cuerpo para formarse una sólida convicción y poder llegar a mencionar si: "la lesión descrita es compatible o no es compatible con el mecanismo de lesión relatado por el paciente".

La hipótesis médica de cómo se produjo la lesión será, entonces, el resultado de la ponderación de la anamnesis, del examen físico y de la presencia o ausencia de signos físicos que respalden el relato del paciente o su acompañante (Bórquez).

Descripción de la lesión. Las lesiones se definen como todo daño o alteración orgánica o funcional de los tejidos del cuerpo, generalmente de origen traumático. Las lesiones son patologías de alta frecuencia y pueden causar desde una incapacidad temporal del afectado, hasta su incapacidad total o, incluso, la muerte (Teke Shilicht, 2004). Pueden ser ocasionadas por agentes biológicos, químicos o físicos. Dentro de estos últimos, los agentes mecánicos son los que constituyen el motivo de consulta más frecuente en la urgencia dental con importancia médico legal, distinguiéndose dos grandes conjuntos de lesiones: contusas y corto-punzantes. Por otro lado, existen otro tipo de lesiones especiales que pudiesen tener que ser evaluadas por odontólogos en urgencia hospitalaria: por proyectil de arma de fuego (PAF) y por mordedura humana (Bórquez).

En el caso de las lesiones contusas encontramos equimosis, hematomas, erosiones, escoriaciones, heridas y fracturas. El mecanismo de producción es la aplicación de energía de manera directa o indirecta con un objeto romo o contra una superficie roma.

Entre las lesiones corto-punzantes tenemos: cortantes o incisas, punzantes o penetrantes, contuso-cortantes y corto-punzantes. Los instrumentos que las provocan pueden tener la punta aguzada y/o borde con filo.

Las lesiones por proyectiles de arma de fuego (PAF) son producto de un mecanismo contuso de alta energía. El proyectil puede rozar, penetrar sin salida, o penetrar con salida. Al rozar, las lesiones pueden producir escoriaciones o heridas superficiales. Al penetrar el PAF, la lesión está constituida por la herida penetrante, con forma más o menos redondeada y un anillo abrasivo y equimótico rodeando los bordes de ésta. Según la distancia del arma de fuego al tejido injuriado, encontraremos hollín, quemaduras, y/o tatuaje en la piel adyacente, que corresponden a escoriaciones producidas por pólvora. Las heridas de salida son también contusas, de un tamaño mayor al de entrada y no contiene los elementos que rodean el orificio de entrada (Saukko \& Knight, 2004).

Las huellas de mordida humana son un patrón representativo de las estructuras dentales sobre un tejido, generalmente, la piel o mucosas. Se puede describir como una lesión compuesta de dos elipses enfrentadas, separadas por un espacio. Cada elipse está conformada por patrones rectangulares hechos por los bordes incisales de los dientes anterosuperiores y anteroinferiores. Ocasionalmente sólo se observa un arco, arcos parciales, y no es poco común encontrar múltiples mordeduras superpuestas. En la periferia de los arcos, se pueden encontrar diversas lesiones (erosiones, escoriaciones, equimosis y/o heridas), mientras que en el centro de la misma, podemos observar sugilaciones producidas por presión negativa de la lengua (American Board of Forensic Odontology, 2015).

Además de las lesiones en tejidos blandos antes descritas, los odontólogos se ven comúnmente enfrentados, en la atención de urgencia, a lesiones de tejido duro maxilofacial. Es fundamental, entonces, que el cirujano dentista esté familiarizado con la nomenclatura actualizada que describa correctamente el Trauma Dentoalveolar (TDA) apreciado, es decir, las lesiones traumáticas que afectan al diente propiamente tal, y a las estructuras de soporte que lo rodean, consecutivas a un impacto violento. En los TDA podemos encontrar lesiones que afecten sólo al diente (fractura coronaria, fractura corono-radicular, fractura radicular), o bien, lesiones que afecten a los tejidos periodontales (concusiones, subluxaciones, intrusiones, extrusiones, luxaciones laterales, avulsiones, fracturas alveolares) (Ministerio de Salud, 2011; Andreasen et al., 2012). 
En Chile, la atención, diagnóstico y tratamiento de los TDA está garantizada por la Ley GES y protocolizada en la Guía Clínica de Atención de Urgencia Odontológica Ambulatoria (Ministerio de Salud, 2011; Ley 19.966: Superintendencia de Salud, 2004).

Es fundamental realizar una correcta descripción y fijación de la o las lesiones observadas, pues muchas veces éstas apuntan al instrumento causal, a las circunstancias en que éstas se produjeron o al autor de las mismas. Si bien hoy en día existen recursos tecnológicos que nos permiten fijar las imágenes, guardando su registro durante mucho tiempo, es fundamental que el profesional maneje un lenguaje adecuado, esté actualizado en la nomenclatura lesionológica vigente y posea capacidad de síntesis de lo observado, pues todo lo anterior debe constar por escrito y debe ser susceptible de ser recordado al momento del juicio oral. Este primer informe es fundamental, pues da cuenta objetiva de la existencia y naturaleza de la lesión, y servirá de base para los posteriores peritajes del lesionado y para la administración de justicia.

Lo anterior es particularmente relevante en el ámbito odontológico, ya que, si bien las lesiones dentoalveolares no conllevan riesgo vital en la mayoría de los casos, es importante establecer las alteraciones funcionales y/o estéticas. La consideración estética a nivel dental es un factor esencial en la calificación de la lesión y en la incapacidad laboral que pueda generar, dependiendo de cuál sea el trabajo desempeñado por el afectado. Cabe considerar también que los dientes, aunque puedan ser rehabilitados con materiales restauradores, nunca recuperan sus características biológicas ni mecánicas originales, lo que debe ser considerado al momento de establecer el pronóstico médicolegal (Contreras \& Hermosilla, 2014). De la misma manera, la evaluación a nivel bucodental por parte del profesional idóneo, es decir, por el cirujano dentista, es imprescindible, ya que las características propias del territorio anatómico, sus patologías y la nomenclatura odontológica son habitualmente desconocidas por otros profesionales de la salud.

Pronóstico médicolegal de la lesión. Al momento de constatar una lesión corporal, el médico debe considerar el pronóstico médicolegal de la misma, es decir, establecer la gravedad de las lesiones en función de la administración de justicia, intentando armonizar sus criterios médicos con los jurídicos (Bórquez). Las lesiones corporales están tipificadas en nuestro Código Penal entre los artículos art. 395 y 403 , tanto en su definición como en la pena para el hechor.
Nuestra jurisdicción establece una clasificación de lesiones que consta de cuatro categorías: las lesiones gravísimas (art. $397 \mathrm{~N}^{\circ} 1 \mathrm{CP}$ ); las lesiones simplemente graves (art. $397 \mathrm{~N}^{\circ} 2 \mathrm{CP}$ ); las lesiones menos graves (art. $399 \mathrm{CP}$ ); y las lesiones leves (art. 494 No 5 CP) (van Weezel, 2008; Código Penal: Fiscalía, Ministerio Público de Chile, 2018). La clasificación clínica de las lesiones se realiza considerando el tiempo que demora en recuperarse la lesión o el tiempo de incapacidad laboral que genera dicha lesión; está dado por el Código Penal (en el caso de lesiones graves y menos graves), y por el uso extendido y aceptado por los expertos (en el caso de las lesiones leves). Si la recuperación demora menos de 15 días en sanar, la lesión es leve; entre 15 y 30 días, menos graves; y más de treinta días, grave. La calificación de lesión "grave gravísima" no responde a un criterio clínico, sino más bien judicial, otorgada por el tribunal cuando, producto de las lesiones sufridas por la víctima, ésta queda "demente, inútil para el trabajo, impotente, impedido de algún miembro importante o notablemente deforme" (Código Penal).

Otras situaciones. Finalmente, cabe mencionar que, además de toda la información anteriormente expuesta, debe quedar registrada en el DAU cualquier situación anexa, como el manejo de evidencias mediante cadena de custodia y la entrega de licencia médica (Bórquez).

En el Sistema de Cadena de Custodia, instaurado con la reforma procesal penal, participan diversas instituciones relacionadas con la impartición de justicia, y, junto a ellas, el Ministerio de Salud. En la red de salud pública, las personas involucradas son los funcionarios y profesionales pertenecientes a las Unidades de Emergencia Hospitalaria, SAPUS u otros lugares de atención tales como Sala de Reanimación, Unidad Clínica Forense Hospitalaria, Box de Atención, Pabellón de Cirugía y en general cualquier dispositivo de salud donde se pueda tomar una muestra y/o evidencia (Minsal, 2015).

La Cadena de Custodia es la secuencia de actos mediante la cual los objetos, documentos, armas o instrumentos, rastros o señales de cualquier clase que parezcan haber estado destinados a cometer el delito y los efectos que de él provengan, son asegurados, trasladados, analizados y almacenados para evitar que se pierdan, destruyan o alteren y así, dar validez a los medios de prueba. Tiene como propósito garantizar la integridad e inalterabilidad de elementos, desde el momento en que son obtenidos del pa- 
ciente, ya sea víctima o imputado, hasta que son entregados a un funcionario de alguna policía, SML o de Fiscalía (Minsal, 2015).

Corolario. La preparación de una correcta constatación de lesiones, o informe médicolegal de lesiones, es un acto médico que no se puede delegar a otro miembro del personal de salud que no sea el profesional médico tratante (médico cirujano o cirujano dentista, general o especialista). Al respecto, Contreras y Hermosilla concluyeron en su estudio del año 2012, que existe un sub-registro de lesiones odontológicas y maxilofaciales en esta primera evaluación del paciente, lo cual determina una falta de elementos para llegar a una correcta administración de justicia, en la aplicación de penas y/o compensación de daños al afectado. Por lo anteriormente expuesto, parece muy necesario instruir a los alumnos de pregrado y actualizar los conocimientos de los cirujano dentistas, en los aspectos no sólo clínico de las lesiones, sino también en los aspectos legales y las implicancias de esta primera evaluación (Contreras \& Hermosilla; LagosTissie et al., 2018).

No podemos perder de vista que en el sistema penal acusatorio que rige en el país desde la instauración completa de la Reforma Procesal Penal, es decir, desde el año 2005, el profesional médico puede jugar distintos roles: imputado (por delitos o cuasidelitos médicos), víctima, testigo presencial o de oídas, perito o testigo experto.

La ley expresa la posibilidad de citar a un juicio oral, en calidad de testigo, al profesional que haya confeccionado un informe médicolegal de lesiones (constatación de lesiones), con el propósito de dar testimonio de manera directa, explicando desde un punto de vista profesional, lo registrado (Código Procesal Penal). Este testimonio estará basado en la constatación inicial que realizó en el lesionado. El papel que juega el cirujano dentista en la impartición de justicia y posibilidad de reparación para aquellas víctimas que llegan al servicio de urgencia donde ejercen sus labores es, entonces, fundamental, ya que cualquier informe de lesiones que se realice posteriormente en el SML, previo a la realización de un juicio penal por lesiones, va a quedar condicionado a la correcta realización de la constatación inicial. Todo cirujano dentista que trabaje en cualquier establecimiento público o privado de salud que pueda recibir pacientes lesionados por cualquiera de las diversas causas ya expuestas, debe tener la capacidad de generar un primer informe de lesiones apropiado a las exigencias legales y éticas que la sociedad exige.
LAGOS, T. D. \& BRAVO, R. L. Elaboration of the forensic report of dental injuries: Recommendations for the general dentist. Int. J. Odontostomat., 13(3):350-356, 2019.

ABSTRACT: The general dentist plays a major role in ascertaining and verifying injuries, especially if he or she, works in an emergency department. The initial evaluation is fundamental for the forensic dentist in order to subsequently state a prognosis of the injuries sustained, indicating how long they will take to heal, and the disability related to time away from employment. Further, it is the forensic dentist's responsibility to evaluate and relate cause of injuries with the criminal offenses being investigated. In this study, updated information about the process is presented of medical injury verification, the injuries report in the Chilean legislation, and the participation of the dentist. The correct preparation of a forensic report of injuries is a medical responsibility that cannot be delegated to another member of the healthcare staff. All dentists must have the ability to generate a first report of injuries, according to society's legal and ethical requirements. The law states that the professional who completes a forensic report of injuries, can be summoned to trial as a witness, with the purpose of providing direct testimony, and objectively explain what he/she observed in that specific case.

KEY WORDS: forensic dentistry, legislation, maxillofacial injuries.

\section{REFERENCIAS BIBLIOGRÁFICAS}

American Board of Forensic Odontology (ABFO). Diplomates Reference Manual. American Board of Forensic Odontology, 2015. Disponible en: http://www.abfo.org/

Andreasen, J. O.; Lauridsen, E.; Gerds, T. A. \& Ahrensburg, S. S. Dental Trauma Guide: a source of evidence-based treatment guidelines for dental trauma. Dent. Traumatol., 28(5):142-7, 2012.

Bórquez, V. P. Elaboración del informe médico de lesiones. Rev. Med. Chile, 140(3):386-9, 2012.

Contreras, C. \& Hermosilla, B. Acreditación de lesiones y trauma dentoalveolar: un desafío. Investig. Forense, 3(1):66-73, 2014.

Fiscalía, Ministerio Público de Chile. Código Penal. Santiago de Chile, Diario Oficial de la República de Chile, 2018.

Fiscalía, Ministerio Público de Chile. Código Procesal Penal. Santiago de Chile, Diario Oficial de la República de Chile, 2000.

Lagos-Tissie, D.; Bravo, R. L; Ramos-Moreno, J. L. \& CioccaGómez, L. Teaching and research in legal dentistry in Chile: current situation and challenges. Int. J. Odontostomat., 12(3):274-9, 2018.

Ministerio de Justicia. Ley 18.290. Ley de Tránsito. Santiago de Chile, Diario Oficial de la República de Chile, 1984.

Ministerio de Justicia. Ley 20.066. Establece Ley de Violencia Intrafamiliar. Santiago de Chile, Diario Oficial de la República de Chile, 2005.

Ministerio de Salud. Decreto Exento 467/13. Aprueba norma técnica $N^{\circ} 149$ sobre estándares de información de salud - 
LAGOS, T. D. \& BRAVO, R. L. Elaboración del informe médicolegal de lesiones odontológico: recomendaciones para el odontólogo. Int. J. Odontostomat., 13(3):350-356, 2019.

Estándar de atención de urgencia de los niveles de alta, mediana y baja complejidad y de la atención primaria. Santiago de Chile, Gobierno de Chile, 2013.

Ministerio de Salud. Guía Clínica AUGE: Urgencias Odontológicas Ambulatorias. Santiago de Chile, Ministerio de Salud, Gobierno de Chile, 2011.

Ministerio del Trabajo y Previsión Social. Ley 16.744. Establece Normas sobre Accidentes del Trabajo y Enfermedades Profesionales. Santiago de Chile, Diario Oficial de la República de Chile, 1984.

Saukko, P. \& Knight, B. Knight's Forensic Pathology. $3^{\text {rd }}$ ed. Londres, Oxford University Press Inc., 2004.

Superintendencia de Salud. Ley 19.966 Establece un Régimen de Garantías Explícitas en Salud. Santiago de Chile, Diario Oficial de la República de Chile, 2004.

Teke Shilicht, A. Lesionología General y Especial. En: Teke Shilicht, A. Medicina Legal y Criminalística. Santiago de Chile, Ediciones Jurídicas de Santiago, 2004. pp.45-139.

van Weezel, A. La Sistemática de los Delitos de Lesiones en el Código Penal y el Régimen Introducido por la Ley $N^{\circ} 20.066$ sobre Violencia Intrafamiliar. Santiago de Chile, Biblioteca de la Defensoría Penal Pública, 2008. Disponible en: http:// www.biblio.dpp.cl/biblio/DataFiles/5267.pdf

\author{
Dirección para correspondencia: \\ Denisse Lagos Tissie \\ Universidad de Chile \\ Santiago \\ CHILE
}

Email: dlagos@odontologia.uchile.cl

Recibido : 29-11-2018

Aceptado: 12-03-2019 